\title{
Single Pole Ground Fault Protection Strategy for MMC-MTDC Based on Voltage Margin Control
}

\author{
Mingguang Zhang ${ }^{1,2,3}$ and Jiawen $\mathrm{Li}^{1,2.3^{*}}$ \\ ${ }^{1}$ School of Electrical and Information Engineering, Lanzhou University of Technology, Lanzhou 730050, P.R. China \\ ${ }^{2}$ Key Laboratory of Gansu Advanced Control for Industrial Processes, Lanzhou University of Technology, Lanzhou 730050, P.R. \\ China \\ ${ }^{3}$ National Demonstration Center for Experimental Electrical and Control Engineering Education, Lanzhou University of \\ Technology, Lanzhou 730050, P.R. China \\ ${ }^{*}$ Corresponding author
}

\begin{abstract}
Modular multi-level converter has become one of the most prospective converter technologies in HVDC transmission. According to the topological structure and mathematical model of MMC, this paper proposes a multi-point DC voltage control strategy based on voltage margin control, and designs the corresponding controller. On this basis, the influence of DC single pole ground fault on the system is analyzed, and the relevant protection strategy is proposed. Finally, the PSCAD/EMTDC is utilized to simulate the single pole ground fault and implement protection strategy. The simulation results indicate that the system can quickly recover after system fault and improve the system stability, the effectiveness of fault protection is proved.
\end{abstract}

Keywords-topological structure; mathematical model of MMC; multi-point DC voltage control strategy; DC single pole ground fault; implement protection strategy

\section{INTRODUCTION}

With the development of power electronic technology, HVDC technology based on voltage source converter has been rapidly developed.[1] Compared with the traditional current source converter, modular multi-level converter (MMC) has a lot of advantages, such as, modular structure, easily expanding, high transmission quality of electricity, and small power transfer loss.[2-4]

With the development and application of MMC-MTDC technology in the multi-terminal DC transmission system, the problem of fault protection has become increasingly important.[5-7] DC fault is one of the most common faults of MMC-MTDC system, that includes bipolar short-circuit fault and single pole ground fault.[8-9] "Handshake principle" of multi-terminal HVDC transmission system for DC bipolar short circuit fault is proposed in [10], and realized the location and isolation of DC fault line. The DC fault clearing and isolation without opening out the AC circuit breakers method is put forward in [11]. A fault protection control strategy of MMC-MTDC with DC bipolar short circuit is analyzed in [12], which can achieve transient and permanent DC short-circuit fault ride-through by changing the control mode. A rapidly identifying method of bipolar permanent fault for MMCMTDC is researched in [13]. In order to consume the unbalanced power that cannot be eliminated by the DC transmission system when the network-side AC system occurs faults, DC side parallel with a unified decentralized small unloading load is described in [14], thus the DC system voltage maintains constant. The robust locally weighted regression approach is used to realize the fast permanent fault identification in transient process in[15].

Aiming at single pole ground fault in MMC-MTDC system, a detailed study has been carried out in this paper. First of all, based on the analysis of half-bridge MMC topology widely used in engineering, the mathematical model of MMC-MTDC is established, and a multi-point DC voltage control strategy based on voltage margin is proposed. Corresponding controller is designed accordingly. Finally, the feasibility of this strategy is verified using PSCAD/EMTDC simulation software.

\section{MULTI-TERMINAL FLEXIBLE DC TRANSMISSION TOPOLOGY AND MATHEMATICAL MODEL}

\section{A. MMC Topology Structure}

Fig. I shows the MMC structure of a three-terminal MMCMTDC power system. It can be seen from the figure that one side of an MMC converter is composed of three phases, and each phase has upper and lower bridge arms. Each bridge arm consists of a inductance, a resistor, and a number of submodule cascading, each sub-module can be used as a switching unit insulated gate bipolar transistor half-bridge (including two IGBTs, two anti-parallel diodes, and a DC energy storage capacitor).

The bridge arm voltage is the sum of the output voltages of all series sub-modules on the upper and lower arms, and the DC voltage is the sum of the upper and lower arm voltages of the same phase. When the full-controlled device $V T_{1}$ turns on and $V T_{2}$ turns off, the sub-module output voltage is the DC capacitor voltage. The sub-module output voltage is zero when $V T_{1}$ turned off and $V T_{2}$ turns on. 


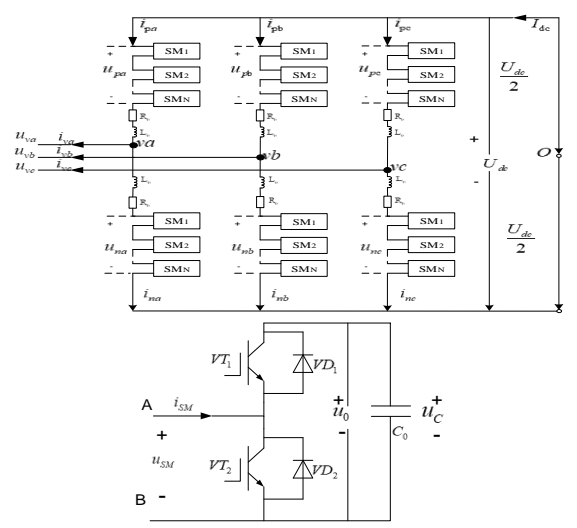

FIGURE I. MMC TOPOLOGY

B. The Mathematical Model of MMC-MTDC System in dq Coordinate

Voltage source converter stations based on MMC's topological structure are connected in parallel to constitute three-terminal MMC-MTDC transmission system, its connection structure shown as in FIG.2-2, $U_{\text {sim }}$ ( $i=a, b, c ; m=1,2,3 ; m$ represents terminal number) is the phase voltage on valve side of ideal transformer on each terminal, $U_{\text {eim }}$ ( $i=a, b, c ; m=1,2,3$ ) is the voltage for the same potential point at connection between reactor of upper and lower arms on each phase and sub-module, $R_{m}(m=1,2,3)$ represents equivalent resistance of AC network on each terminal, $L_{m}$ represents equivalent reactance after leakage reactance inductance of transformer $L_{T}$ and reactance inductance of arm ${ }^{L_{0}}$ are connected in series, its expression is:

$$
L_{m}=L_{T}+\frac{L_{0}}{2}
$$

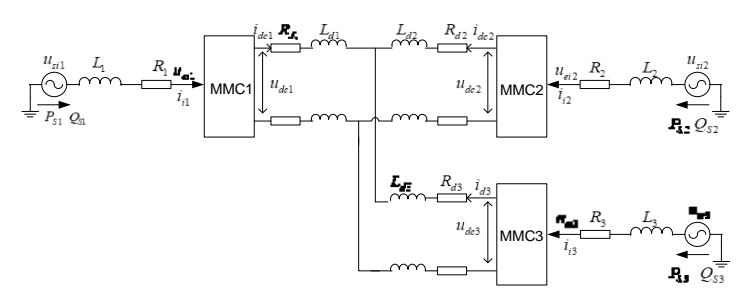

FIGURE II. CONNECTION STRUCTURE OF MMC-MTDC

According to the KVL and KCL circuit laws. The expression of the AC side of each converter station of a threeterminal flexible DC transmission system can be obtained:

$$
L_{m} \frac{d I_{m}}{d t}=-R_{m} I_{m}+\left(U_{s m}-U_{e m}\right)
$$

In the above formula:

$$
I_{m}=\left[\begin{array}{lll}
i_{a m} & i_{b m} & i_{c m}
\end{array}\right]
$$

$$
\begin{gathered}
U_{e m}=\left[\begin{array}{l}
u_{e a m} \\
u_{e b m} \\
u_{e c m}
\end{array}\right]=\left[\begin{array}{c}
K_{m} U_{d c m} \cos \left(\omega t+\delta_{m}\right) \\
K_{m} U_{d c m} \cos \left(\omega t-\frac{2 \pi}{3}+\delta_{m}\right) \\
K_{m} U_{d c m} \cos \left(\omega t+\frac{2 \pi}{3}+\delta_{m}\right)
\end{array}\right] \\
U_{s m}=\left[\begin{array}{l}
u_{s a m} \\
u_{s b m} \\
u_{s c m}
\end{array}\right]=\left[\begin{array}{c}
U_{s m} \cos \omega t \\
U_{s m} \cos \left(\omega t-\frac{2 \pi}{3}\right) \\
U_{s m} \cos \left(\omega t+\frac{2 \pi}{3}\right)
\end{array}\right]
\end{gathered}
$$

where $K_{m}$ represents utilization factor of DC voltage; $\delta_{m}$ represents the angle between input voltage of converter station and system voltage.

Then transform formula (2) into dq coordinate:

$$
\frac{d}{d t}\left[\begin{array}{l}
i_{d m} \\
i_{q m}
\end{array}\right]=\frac{1}{L_{m}}\left[\begin{array}{cc}
-R_{m} & \omega L_{m} \\
-\omega L_{m} & -R_{m}
\end{array}\right]\left[\begin{array}{c}
i_{d m} \\
i_{q m}
\end{array}\right]+\frac{1}{L_{m}}\left[\begin{array}{c}
U_{s d m}-U_{s q m} \\
U_{s q m}-U_{e q m}
\end{array}\right]
$$

Accordingly, the expression of voltage and current on DC side of three-terminal MMC-MTDC system is:

$$
\begin{gathered}
U_{m}=u_{d c m}-L_{d m} \frac{d i_{d c m}}{d t}-i_{d c m} R_{d m} \\
U_{1}=U_{2}=U_{3} \\
i_{d c 1}+i_{d c 2}+i_{d c 3}=0
\end{gathered}
$$

where $U_{m}$ is the equipotential voltage at parallel connection of DC lines in three-terminal DC system. Due to DC current has little effects, therefore,

$$
L_{d m} \times \frac{d i_{d c m}}{d t} \approx 0
$$

The active power and reactive power infusing from AC side into converter are respectively:

$$
\begin{gathered}
P_{s m}=\frac{3}{2}\left(u_{s d m} i_{d m}+u_{s q m} i_{q m}\right) \\
Q_{s m}=\frac{3}{2}\left(u_{s d m} i_{q m}-u_{s q m} i_{d m}\right)
\end{gathered}
$$

Under voltage balance condition of three-phase network, the vector direction of voltage is $d$ axis direction, and 
$U_{s d}=U_{s}$ ( $U_{s}$ is the voltage amplitude of AC system), $U_{s q}=0$, then above equation can be simplified into:

$$
\begin{gathered}
P_{s m}=\frac{3}{2} U_{s m} i_{d m} \\
Q_{s m}=-\frac{3}{2} U_{s m} i_{q m}
\end{gathered}
$$

Power of DC side of MMC-MTDC is:

$$
P_{d m}=u_{d c m} i_{d c m}
$$

Without considering resistor resistance and active loss of convertor, the power absorbed by convertor from AC network is equal to power transmitted by convertor to DC network, then:

$$
\frac{3}{2} U_{s m} i_{d c m}=u_{d c m} i_{d c m}
$$

(6)-(9) and (14) are the mathematical model of threeterminal flexible DC transmission in $d q$ coordinate system.

\section{MMC-MTDC CONTROL STRATEGY RESEARCH}

\section{A. Based on the Voltage Margin of Multi-Point DC Voltage Control Principle}

The multi-point DC voltage control strategy based on voltage margin control is adopted for three-terminal MMC flexible DC transmission system. The power infused from AC side to DC side is selected as positive, then its operating characteristics are as shown in FIG.3-1. $U_{d \max }$ and $P_{H r e f}$ are respectively upper and lower limits of DC voltage. $U_{\text {eim }}$ and $P_{\text {Lref }}$ are respectively upper and lower operational limits of active power. $U_{\text {dref }}$ is expected operational voltage of convertor, with some margin kept. Assuming the three-terminal MMC-MTDC system is connected according to FIG.2-2, then the relation between unknown parameter $\left(U_{d c 2}, U_{d c 3}\right.$ and $\left.P_{1}\right)$ on each terminal and known parameter is:

$$
\begin{gathered}
u_{d c}=u_{d c 1}-R_{d 1} i_{d c 1}+R_{d c 2} i_{d c 2} \\
u_{d c 3}=u_{d c 1}-R_{d 1} i_{d c 1}+R_{d 3} i_{d c 3} \\
P_{1}=P_{2}+R_{d 2}\left(i_{d c 2}\right)^{2}+P_{3}+R_{d 3}\left(i_{d c 3}\right)^{2}
\end{gathered}
$$

Above analysis shows: when the DC line is too long, the voltage drop and power loss caused by resistance of DC line leads to some deviation at operation point. The system changes from originally operating point $A$ to point $B$ as shown in the FIG.3-1. The power transmitted by station 1 is higher than the sum of the power absorbed by the other two stations, while the DC voltage for station 2 and station 3 is lower than the reference value of DC voltage for station 1 . The condition to maintain system stability is the voltage reference value of station 2 being lower than voltage of actual operating point, now the DC voltage is controlled by station 1 . However, if the value of voltage margin for station 2 is set as a small one, which makes the voltage reference value higher than the actual operating voltage after operation, then station 1 and station 2 are in the mode of constant DC voltage simultaneously, in such cases, convertor station 2 loses the power control capacity, and the system operates at point $\mathrm{C}$ in the figure. Now the system has poor stability. Thus, the line resistance does not only lead to line drop and line loss, but also results in some differences between actual operational voltage parameter and set reference value, and influences the stability of margin control of DC voltage and setting of voltage margin.

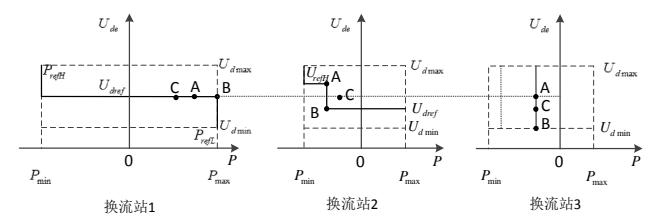

FIGURE III. VOLTAGE MARGIN CONTROL

\section{B. Controller Design}

The master and slave controllers of three-terminal MMC flexible DC transmission system all adopt multi-point DC voltage control strategy based on voltage margin control, which has high adaptability in current response and internal current limiting capacities. The outer control generates designated value of internal-loop current according to given DC voltage, active power and reactive power, while the inner current control loop regulates the output voltage of convertor to make the actual current value $i_{d}$ and ${ }^{i_{q}}$ quickly track its designated value $I_{d r e f}$ and $I_{\text {qref }}$. As pressure drop of line influences the voltage margin to a certain extent, the selection equation of voltage margin $\Delta U$ is obtained as follows:

$$
\Delta U=\sum_{j=1}^{n} r_{j} l_{j} \cdot i_{d c m}+k U_{d c r e f}=U_{d r o p}+\Delta
$$

where ${ }^{r_{j}}$ represents resistance for unit length of DC overhead line; ${ }^{l}{ }_{j}$ is length of line; ${ }^{j}$ is the different conductor segments between two terminals of required voltage margin; $m$ is terminal number of convertor station, $U_{d r o p}$ is voltage drop of DC line between slave station and master station, which is closely related to line length and DC, $U_{d c r e f}$ is reference value of DC voltage for master convertor station; $\Delta$ is control voltage correction, being generally small. $\mathrm{K}$ is proportional constant, being $1 \%-5 \%$, The following FIG.3-2 shows the controller structure: 


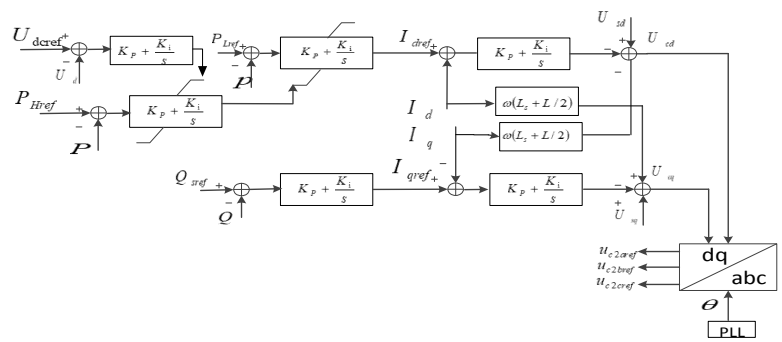

FIGURE IV. CONTROLLER STRUCTURE

\section{Single POLE GROUND FAULT PROTECTION}

\section{A. The Way of the DC Side Grounding}

MMC adopts structure of tandem sub-modules, the energystorage capacitor is independently and evenly dispersed in submodules, and the neutral grounding terminal of capacitor cannot be led out conveniently, thus MMC is more suitable to adopt the mode of large clamping resistance to ground the DC side, as shown in FIG.4-1, in the case of single pole ground fault, the ground branch, DC system and AC system do not constitute a discharge circuit, but only change the position of potential reference point of DC transmission system and do not need to turn off convertor station, the system can continue to run. However, as the voltage increase for the complete terminal is two times of the nominal value, the insulation for convertor valve and other equipment should be able to meet a withstand voltage level.

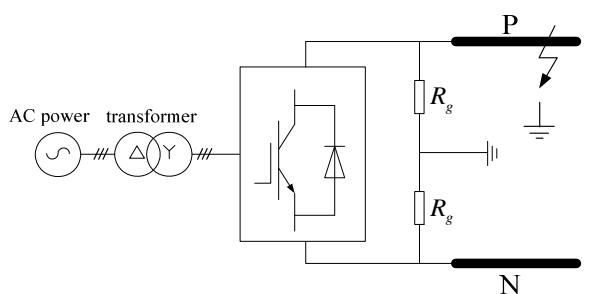

FIGURE V. SCHEMATIC DIAGRAM OF THE SINGLE POLE TO GROUND FAULT UNDER THE DC SIDE GROUNDING MODE

\section{B. Single Pole Ground Fault Protection Strategy}

A method of fault location control and protection is designed for single pole ground fault. The method cannot only locate and isolate faulty line, but also has the function of reclosing after fault occurs, which can judge the temporary fault and permanent fault to process them differently. The control and protection strategy of single pole ground fault is as follows concretely: Firstly the fault type is judged according to fault signature to determine whether single pole ground fault occurs; if yes, each convertor station is quickly turned off and the breaker on AC side of convertor station is disconnected; when the current on DC side is lowered to 0 , the DC switches on two ends of all lines are quickly disconnected, to make the DC switch on terminal of line OFF, then the quick DC switch at head terminal of each line is closed and convertor is unlocked, to verify the live part of each convertor station port. If the absolute value of bipolar DC voltage is lower than the judged voltage, this can serve as basis to judge that the singlepole ground fault in the connected DC line still exists, or the fault can be regarded as having been eliminated. If single-pole ground fault has been eliminated, the DC switch at terminal of all lines is closed and breaker on AC side of convertor station is closed too to restore normal power supply by multi-terminal system. If single-pole ground fault still exists, the convertor station on faulty line side is turned off, and the quick DC switch at head terminal of this faulty line is disconnected to isolate the faulty line. When the DC voltage for the port of convertor station on non faulty line side reaches the pre-set value, the DC switch on terminal of non faulty line is quickly turned off, lastly, the AC circuit breaker for convertor station on non faulty line side is turned off, and the rest multi-terminal system can still continue to run.

\section{SIMULATION VERIFICATION}

\section{A. Parameter Determination}

The simulation model of flexible DC transmission system was built in PSCAD environment for verification, with connection mode as shown in FIG.2-2. The three convertor stations all adopt MMC topological structure with 21-level. The system adopts multi-point DC voltage coordinated control strategy based on voltage margin control. As the master convertor station, the convertor station 1 has reference voltage $U_{\text {def }}$ of $400 \mathrm{kv}$ in good running, the upper and lower limits PHref and PLref of active power are respectively set as 100MW and-100MW. The convertor station 2 is the slave convertor station, whose Udef is $400 \mathrm{kv}$, PHref and PLref are respectively 26MW and-26MW. The convertor station 3 connects to passive network. Assuming the DC side of convertor station 1 has ground fault at 2 s, with fault duration being $0.1 \mathrm{~s}$, then, the simulative waveform of system is as shown in Fig.4-1. The parameters of convertor station are shown in table 1 :

TABLE I. MMC CONVERTER STATION PARAMETERS

\begin{tabular}{|c|c|c|}
\hline parameter & $\begin{array}{c}\text { Labe } \\
1\end{array}$ & $\begin{array}{c}\text { Referen } \\
\text { ce }\end{array}$ \\
\hline The sampling period & $T_{s}$ & $50\left(u_{s}\right)$ \\
\hline Number of sub-modules & $N$ & 20 \\
\hline DC line voltage & $U_{d c}$ & $\begin{array}{l}400( \\
K V)\end{array}$ \\
\hline Sub-module capacitance & $C$ & $\left.\begin{array}{c}12( \\
m F\end{array}\right]$ \\
\hline AC system voltage & $U$ & $\begin{array}{l}420( \\
K V)\end{array}$ \\
\hline $\begin{array}{c}\text { AC system equivalent } \\
\text { inductance }\end{array}$ & $L$ & $\begin{array}{c}26 \\
(m H\end{array}$ \\
\hline Transformer ratio & $k$ & $420 / 230$ \\
\hline Bridge arm inductance & $L_{\text {arm }}$ & $\begin{array}{c}40( \\
m H)\end{array}$ \\
\hline $\begin{array}{c}\text { Sub-module capacitor voltage } \\
\text { reference }\end{array}$ & $U_{c}$ & $\begin{array}{c}20( \\
K V)\end{array}$ \\
\hline Sub-module clamp resistance & $R_{g}$ & $\begin{array}{c}100 \\
(\Omega)\end{array}$ \\
\hline
\end{tabular}




\section{B. Single Polar Ground Fault Characteristics}

When the system detected single-pole ground fault at 2s, the control and protection strategy of single pole ground fault is adopted: the three-terminal convertor station was turned off 0.01s later, the three-terminal AC circuit breaker was tripped 0.08 s later to cut off feed-in of AC, when the DC at 2.15s lowered to 0 , all quick DC switches of multi-terminal system are disconnected. The quick DC switch at terminal of line is kept off at 2.16s, then the quick DC switch at head terminal of line is closed and the convertor station is unlocked to verify the live part. FIG.5-2 shows that the absolute value of DC voltage on positive pole and negative pole of three-terminal convertor station is all higher than the voltage criteria, so it can be judged that the fault has been eliminated. Thus, the quick DC switch on terminal of each line is closed at $2.17 \mathrm{~s}$, and breaker on AC side of each convertor station is switched on at $2.24 \mathrm{~s}$, thereby finishing restarting of multi-terminal system. The current waveforms of positive and negative lines for three-terminal convertor station are basically the same, thus, this paper only lists the waveform of convertor station 1. Fig. 5-1 shows that the capacitive voltage of sub-module for convertor station 1 is fluctuated, and restored after fault clearance; the DC voltage and active power also got right immediately after influenced by the fault. For the AC system, the AC voltage on valve side of convertor station deviates, with amplitude generally falling, as shown in FIG.5-4. As the DC side grounds via clamp resistance, the capacitive voltage did not change largely during fault duration. When the fault was cleared, the voltage to earth of positive and negative of DC side could be restored.

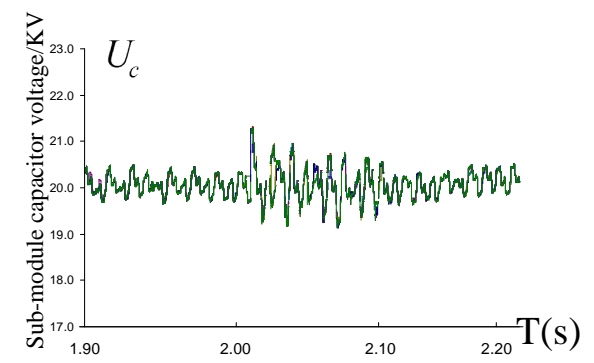

FIGURE VI. CONVERTER STATION 1 SUB-MODULE CAPACITOR VOLTAGE

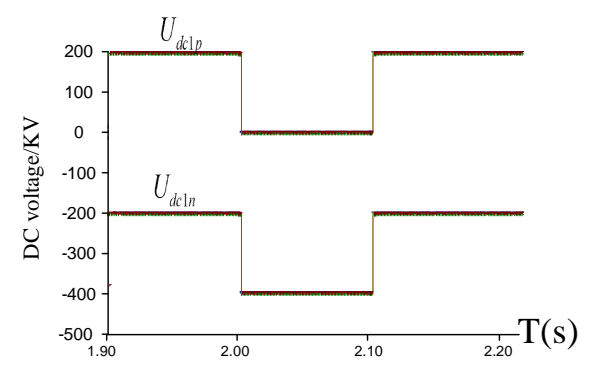

FIGURE VII. TWO POLES OF CONVERTER STATION 1 DC VOLTAGE

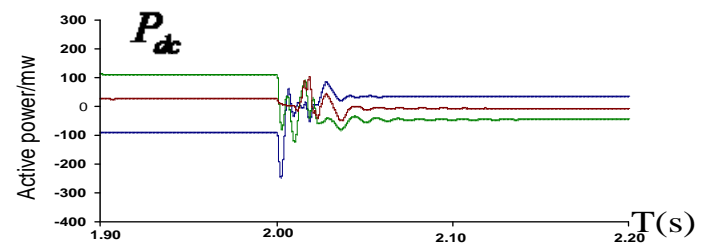

FIGURE VIII. ACTIVE POWER AT EACH CONVERTER STATION

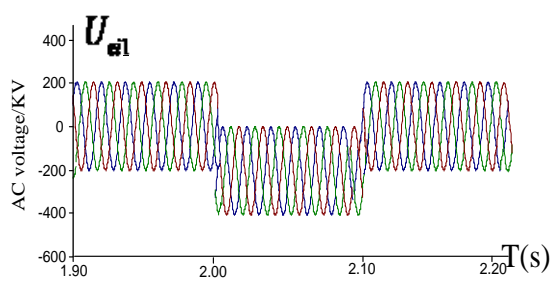

FIGURE IX. CONVERTER 1 VALVE SIDE AC VOLTAGE

\section{CONCLUSION}

According to the MMC-MTDC system, this paper analyzes the DC single pole ground fault characteristics of and protection strategies.

(1) The MMC-MTDC DC side fault was studied from the topological structure of MMC, and the mathematical model under the dq axis was analyzed to prepare for the follow-up study.

(2) According to the characteristics of its mathematical model, a multi-point DC voltage control strategy based on voltage margin is designed for MMC's flexible dc transmission system. After the control strategy is adopted, the multi-terminal system can automatically switch the control mode after the fault disturbance, and improve the fault ride-through ability of the multi-terminal system.

(3) When the MMC-MTDC has a single pole ground fault, the multi-terminal system can still transmitted power normally due to the MMC's own properties.

The theory analysis and the proposed strategy are demonstrated by the PSCAD/EMTDC simulation.

\section{ACKNOWLEDGMENT}

The author gratefully acknowledge the financial support from the National Natural Fund Project of China under Grant 51567016.

\section{REFERENCES}

[1] Xu Zheng, Xue Yinglin,Zhang Zheren VSC-HVDC technology suitable for bulk power overhead line transmission[J].Proceeding of the CSEE,2014,34(29):5051-5062.

[2] Ma Weimin, Yang Yiming, Flexible HVDC Transmission Technology's Today and Tomorrow.[J] High Voltage Engineering, 2014,40 (8); 24292439.

[3] Xiao Gangqing, Xu Zheng, Xue Yinglin, Start Control Strategy of MMC-MTDC system.[J] .High Voltage Engineering, 2014,40 (8): 25502557.

[4] Li Yaman, Jiang Weiyong, Yu Shifeng, System Design of ZhouShan Multi-terminal VSC-HVDC Transmission Poject.[J] High Voltage Engineering, 2014,48 (8): 2490-2496. 
[5] Chaudhun N, Chaudhun B, Adaptive droop control for effective power sharing in multi-terminal DC (MTDC) grids [J]. IEEE Transactions on Power Systems, 2013, 28 (1): 21-29.

[6] Beerten J, Cole S, Belmans R, Modeling of multiterminal VSC-HVDC systems with distributed DC voltage control [J]. IEEE Transactions on Power Systems, 2013, 29 (1): 34-42.

[7] Wang Y, Marquardt R. Future HVDC-grids employing modular multilevel converters and hybrid DC-breakers [C] // European Conference on Power Electronics and Applications (EPE), Lille, France, 2013: 1-8.

[8] Liang Yingyu, Zhang Tao, Liu Jianzheng, A Circulating Current Suppressing Method for Modular Multilevel Converter Under Unbalanced Grid Voltage [J] .Transactions of China Electrotechnical Society, 2016,31 (9): 120-128.

[9] Liao Wu, Huang Shoudao, Huang Sheng, Unbalanced Grid Fault Ridethrough Control Method of HVDC Power Transmission Based on Modular Multilevel Converters.[J] Transactions of China Electrotechnical Society, 2015,30 (12): 197-203.

[10] Tang Lianxiang, Ooi B T, Locating and isolating DC faults in multiterminal DC systems [J]. IEEE Trannsactions on Power Delivery, 2007, 22 (3): $1877-1884$

[11] ZHAO Chengyong, XU Jianzhong, LI, A Hybrid HVDCSystem With DC Fault Ride-through Capability.[J]. proceedings of the CSEE.2015,43 (17): 4345-4352.

[12] Wang Shanshan, Zhou Xiaoxin, Tang Guangfu, Analysis of Submodule Overcurrent Caused by DC Pole-to-Pole Fault in Mudular Multilevel Converter HVDC System.[J] Proceeding of the CSEE, 2011,31 (1): 1-7.

[13] Luo Yongjie, Li Yaohua, Li Zixin, DC Short-Circuit Fault Ride-through Control Strategy of Full-Brige MMC-HVDC Systems [J] .Proceedings of the CSEE, 2016,36 (7): 1933-1943.

[14] Chang Fei,Wang Yi,Wang Zhen Yi,XU Shu Kai,Faults Feature Analysis and Control Protection Strategies of Modular Mutilevel Converter Based High Voltage Direct Current.[J],High Voltage Engineering,2015,41(7):2428-2434.

[15] Wang Shuai, Bi Tian Shu,Li Wei,Jia Ke, A fast bipolar permanent fault identification approach for MMC-MTDC lines.[J] Rennewable Energy Resources,2017,35(1):43-49. 